University of South Carolina

Scholar Commons

8-22-1983

\title{
Magnetic Field Behavior of a Josephson-Junction Array: Two- Dimensional Flux Transport on a Periodic Substrate
}

\author{
Richard A. Webb \\ University of South Carolina - Columbia, webbra@mailbox.sc.edu \\ Richard F. Voss \\ G. Grinstein \\ P. M. Horn
}

Follow this and additional works at: https://scholarcommons.sc.edu/phys_facpub

Part of the Physics Commons

Publication Info

Published in Physical Review Letters, ed. Gene D. Sprouse, Volume 51, Issue 8, 1983, pages 690-693. Webb, R. A., Voss, R. F., Grinstein, G., \& Horn, P. M. (1983). Magnetic field behavior of a JosephsonJunction array: Two-dimensional flux transport on a periodic substrate. Physical Review Letters, 51(8), 690-693. DOI: 10.1103/PhysRevLett.51.690

(C) Physical Review Letters, 1983, American Physical Society

This Article is brought to you by the Physics and Astronomy, Department of at Scholar Commons. It has been accepted for inclusion in Faculty Publications by an authorized administrator of Scholar Commons. For more information, please contact digres@mailbox.sc.edu. 


\title{
Magnetic Field Behavior of a Josephson-Junction Array: Two-Dimensional Flux Transport on a Periodic Substrate
}

\author{
Richard A. Webb, Richard F. Voss, G. Grinstein, and P. M. Horn \\ IBM Thomas J. Watson Research Center, Yorktown Heights, New York 10598
}

(Received 14 January 1983)

\begin{abstract}
This Letter reports measurements down to $3 \mathrm{mK}$ of the magnetic field dependence of the resistance and critical current of a weakly coupled periodic array of Josephson junctions. Below $700 \mathrm{mK}$ the resistance is an oscillatory function of field. The temperature dependence of the resistance for integral numbers of flux quanta per cell suggests the existence of a Kosterlitz-Thouless transition involving the unbinding of defects in the flux lattice.

PACS numbers: $74.50 .+r, 05.70 . \mathrm{Fh}, 74.60 . \mathrm{Ge}$
\end{abstract}

The pioneering ideas of Kosterlitz and Thouless $^{1}$ have stimulated considerable theoretical work $^{2-8}$ on the problems of vortex unbinding and melting in two dimensions (2D) on both smooth and periodic substrates. The bulk of the experimental work ${ }^{9-11}$ has considered the resistive transition in thin-film granular superconductors in terms of vortex unbinding on a smooth substrate. Similar measurements ${ }^{12}$ in the presence of a magnetic field are suggestive of (smooth substrate) flux-lattice melting. Recent experiments on Josephson junction ${ }^{13}$ and proximity-coupled superconducting ${ }^{14,15}$ arrays have also provided some verification of vortex-unbinding predictions. These arrays can be more homogeneous than other experimental systems and the relevant parameters can be directly measured or calculated. As shown in this Letter, however, it is the spatial periodicity of the array that leads to dramatic new effects not possible on a smooth substrate.

As background, an idealized infinite superconducting film can support magnetic flux only in quantized vortices. Under the influence of the Lorentz force from a driving current, vortex motion gives rise to a finite voltage and nonzero resistance $R$. Although the net flux must vanish in zero magnetic field, equal numbers of positive and negative thermally excited vortices can be present. At low $T$ these vortices exist only as bound pairs and, hence, $R=0$. Finite driving currents or higher temperatures cause some pairs to unbind and lead to $R>0$. A magnetic field $H$ introduces vortices of one polarity in addition to any thermally excited ones. In the absence of thermal excitation and substrate potential, the mutual repulsion of these vortices would lead to a regular lattice with spacing determined solely by $H$. In arrays, however, this natural periodicity interacts with the periodic substrate potential and, as with charge density waves, leads to commensurate-incommensurate transport effects as $H$ is varied. The fundamental periodicity with $\boldsymbol{H}$ has been observed in many modulated superconducting structures ${ }^{13,14,16}$ and is qualitatively well understood. When the natural flux spacing is commensurate with the substrate there is a large energy barrier for transport. At other spacings the vortex-vortex repulsion competes with the substrate potential and the effective barrier is lowered. In this Letter, we present new array measurements which suggest that the transport properties at commensurate values of $H$ are dominated by defects (positive and negative vacancies) in the flux lattice. Since these defects interact with the logarithmic 2D Coulomb potential, we expect a KosterlitzThouless (KT) transition at which the defect pairs unbind.

The fabrication details of the weakly coupled all- $\mathrm{Nb}$ array have been previously reported. ${ }^{13}$ As shown in Fig. 1(a), the basic structure consists of two types of superconducting grains; squares and crosses. Whenever a cross overlaps a square a $1 \mu \mathrm{m}$ by $1 \mu \mathrm{m}$ Josephson junction is formed. The fundamental cell is the smallest unit surrounded by a loop containing four Josephson junctions and is $64 \mu \mathrm{m}^{2}$ in area. The entire $0.8 \times 0.8-\mathrm{mm}^{2}$ array contains 20000 junctions and 10000 such cells. The capacitance of each junction is $0.1 \mathrm{pF}$ and the normal-state resistance is $50000 \Omega$. Four-terminal $I-V$ measurements were made on the sample which was placed inside the mixing chamber of a dilution refrigerator in a shielded nonmagnetic environment.

In zero magnetic field, measurement $\mathrm{s}^{13}$ of the maximum apparent critical current $I_{c}$ of the entire array correspond to a minimum vortex-unbinding temperature $T_{\mathrm{KT}}$ of $15 \mathrm{mK}$. Although the zero-bias resistance $R_{0}(T)$ fits the expected form over a decade in temperature with this $T_{\mathrm{KT}}$, de- 


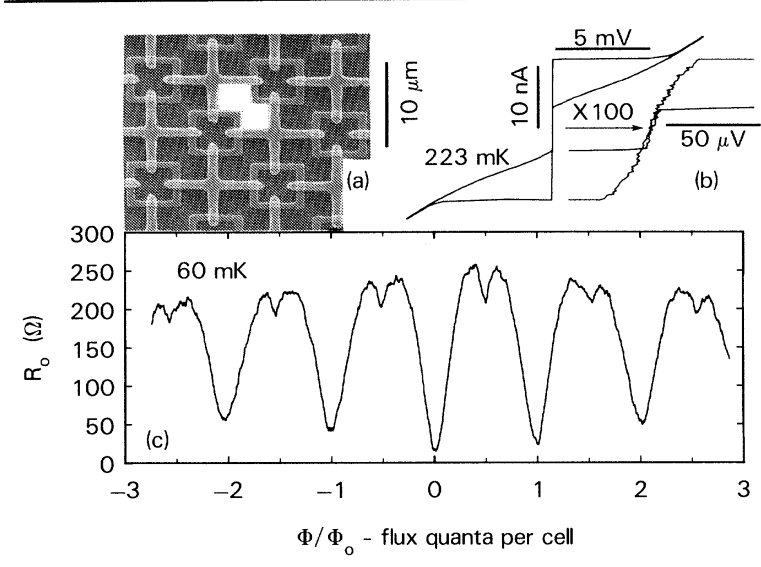

FIG. 1. (a) Micrograph of array structure with a unit cell highlighted. (b) $I-V$ curve of the array at $223 \mathrm{mK}$ and expanded voltage scale showing flux flow resistance $R_{0}$. (c) $R_{0}(H)$ in low fields at $60 \mathrm{mK}$.

viations attributable to finite-size effects ${ }^{17}$ are observed at low $T$. Concomitantly, the $I-V$ curves never show the expected ${ }^{2}$ nonlinear behavior near $T_{\text {KT }}$ because of current-induced pair breaking. Instead, as shown in Fig. 1(b), the $I-V$ curves below $400 \mathrm{mK}$ appear similar to those for a single junction with $I_{c}$ defined by an abrupt switch to a much higher resistance. For sufficiently small currents, $I<I_{c} / 2$, the $I-V$ curves of our array are quite linear and characterized by a well defined $R_{0}$. We believe that these low-T linearities are also due to the thermally generated free vortices present in any finite sample. The magnitude of this flux-flow resistance $R_{0}$ is proportional to the number of free vortices times their mobility. The $I-V$ linearities suggest that the vortex pinning energy to the array structure, which dominates the mobility, is small compared to thermal energies. Moreover, the persistence of this linearity in an applied $H$ suggests that the transport continues to be controlled by the motion of individual vortices.

Figure 1(c) demonstrates the fundamental oscillatory behavior of $R_{0}$ with $H$ at $60 \mathrm{mK}$. For all $R_{0}(H)$ measurements reported here, $I \simeq I_{c} / 10$. $R_{0}(H)$ is periodic in $\varphi / \varphi_{0}$ where $\varphi$ is the average flux in each cell of the array and $\varphi_{0}$ is the flux quantum $h / 2 e$. The resistance minima at integer multiples of $\varphi_{0}$ per cell occur when the natural flux periodicity is commensurate with the lattice pinning structure and any vortex motion becomes more difficult. Although this fundamental periodicity is common in modulated structures, ${ }^{13,14,16}$ our array shows for the first time a new local minimum in $R_{0}(H)$ at $\psi=\left(N+\frac{1}{2}\right) \psi_{0}$. We interpret

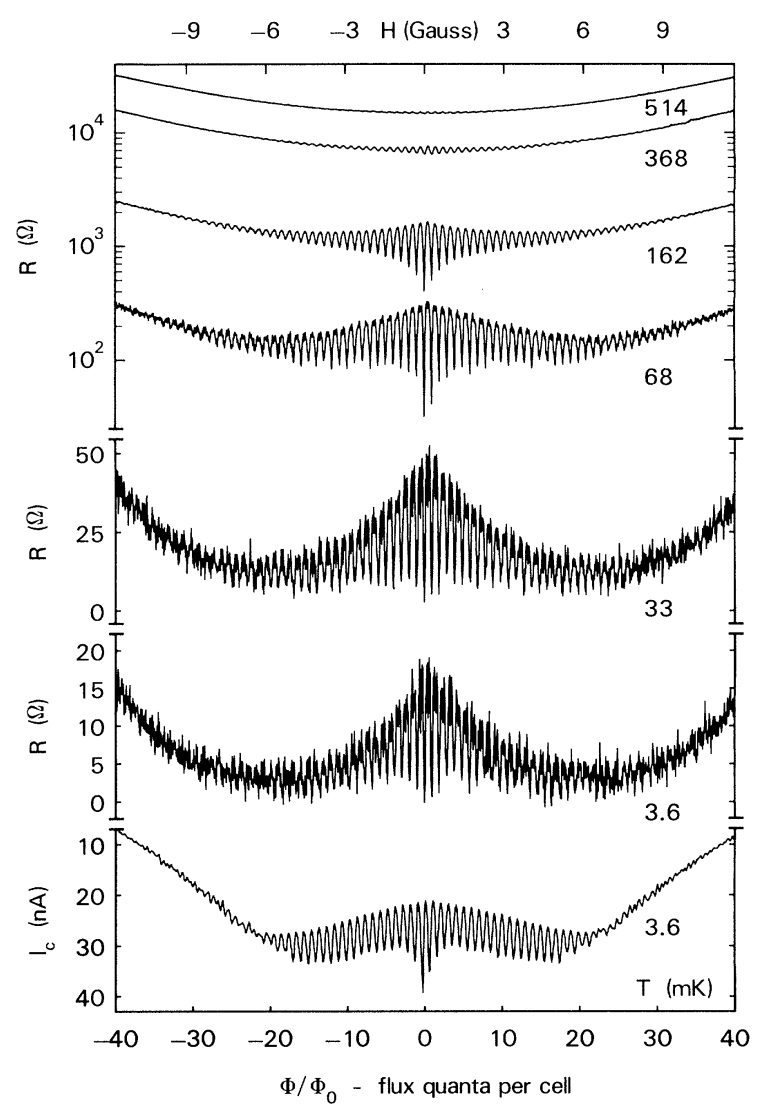

FIG. 2. $R_{0}(\varphi)$ at large fields at various temperatures. Bottom trace shows $I_{c}(\varphi)$ at $3.6 \mathrm{mK}$.

this behavior, which is only visible when $T<170$ $\mathrm{mK}$, as the formation of a commensurate superlattice with alternating cells of $N$ and $N+1$ flux quanta. This superlattice is not observed if the array is either cooled too rapidly through the $\mathrm{Nb}$ $T_{c}$ or cooled in a large $H$ gradient. Consequently, we believe that the superlattice formation indicates that the array itself is fairly homogeneous. The formation energy of even higher-order commensurate structures at $\varphi=N \varphi_{0} / M$ should decrease as the order $M$ increases and the resistance minima should be smaller and visible only at lower $T$. Although $R_{0}(H)$ does develop additional reproducible structure below $40 \mathrm{mK}$, evidence for specific higher-order structures is inconclusive. Recent work ${ }^{7}$ on a frustrated $X-Y$ model provides a theoretical basis for the $N \varphi_{0}$ periodicity and suggests that the $\varphi_{0} / 2$ superlattice should exhibit a phase transition where $R_{0}\left(\varphi_{0} / 2\right) \rightarrow 0$ at some $T<T_{\mathrm{KT}}$.

Figure 2 illustrates the remarkable extent to which these oscillations persist to high $H$ over a wide range of $T$. Below $700 \mathrm{mK}$, the fundamental 
periodicity begins to develop at low $H$ and, as $T$ is lowered, extends progressively to higher $H$. The $\left(N+\frac{1}{2}\right) \varphi_{0}$ superlattice is barely visible in Fig. 2 but extends beyond $25 \varphi_{0}$ at the lowest $T$. At constant $\boldsymbol{H}, R_{0}(T)$ drops rapidly with decreasing $T$, while at constant $T, R_{0}(H)$ eventually increases with increasing $H$ as a result of the large number of field-induced vortices. The bottom trace in Fig. 2 shows $I_{c}(H)$ at $3.6 \mathrm{mK}$ and demonstrates that the relation between $R_{0}(H)$ and $I_{c}(H)$ is similar to that for a single junction; as the effective barrier $I_{c}$ increases, the rate of thermally excited flux transport $R_{0}$ across the barrier decreases. There are, however, detailed differences. Here, the apparent $I_{c}$ characterizes the rapid avalanche of flux simultaneously across the entire array $\left(I_{c}\right.$ is the single-junction critical current $i_{c}$ times the number of junctions across the array). $R$, on the other hand, characterizes the slow transport of individual quanta (possibly one barrier at a time) with small driving force.

The most unexpected feature in Fig. 2 is the development below $50 \mathrm{mK}$ of minima in the lower envelope $\left(\varphi=N \varphi_{0}\right)$ for $|H| \simeq 5-7 \mathrm{G}\left[(15-25) \varphi_{0}\right]$. This effect has not been seen in other modulated structures. Surprisingly, below $30 \mathrm{mK}, R_{0}\left(N \varphi_{0}\right)$ in this region is very nearly equal to the resistance at $H=0$ and almost zero. Naively, one expects an $R_{0}(H)$ at all $T$ similar to that shown at $162 \mathrm{mK}$; at large $H$ the amplitude of the oscillations should decrease (partly as a result of inhomogeneities) and approach the familiar fluxflow resistance $R_{0} \propto H$. For the range of $H$ shown in Fig. 2, we expect little change in the individual junction's $i_{c}$. Since the $N \varphi_{0}$ commensurate minima require both a substrate potential (varying as $i_{c}$ ) and a vortex-vortex repulsive interaction, the different behavior of different minima thus reflects the importance of the vortex-vortex interaction. We do not have a qualitative explanation for the existence of a minimum in $R_{0}\left(N \varphi_{0}\right)$. Presumably its position and amplitude are sensitive functions of the substrate potential and vortex interactions (including dielectric screening by the commensurate background) in this particular array.

It is, however, possible to understand qualitatively the temperature dependence of $\boldsymbol{R}_{0}\left(N \varphi_{0}\right)$ within the following physical framework. At $\varphi=N \varphi_{0}$, the ground state corresponds to exactly $N \varphi_{0}$ in each cell. As the temperature is raised, defects can be thermally generated. These defects are positive and negative vacancies (corresponding to $N+1$ and $N-1$ flux quanta in nearby cells) simi- lar to the vortex-antivortex pairs in zero field. The energy necessary to create a defect pair at large separation, $\Delta E \propto(N+1)^{2}+(N-1)^{2}-2 N^{2}$, is independent of $N$. Unlike the smooth-substrate limit, however, the remaining vortices have commensurate long-range order imposed by the substrate. This flux lattice remains pinned to the array structure and it is only the free defects that move with a weak driving field. In the language of the Coulomb gas, the commensurate flux lattice can lead to only dielectric screening. Consequently, the low- $T$ interaction between defects is logarithmic in their separation and at some critical temperature $T_{c}$ there should be a Kosterlitz-Thouless unbinding transition. This scenario predicts that the behavior of $R_{0}\left(N \varphi_{0}\right)$ should be similar to the behavior at $H=0$ except that the transition temperature could depend on $N$. Thus, the local minimum in $R_{0}\left(N \varphi_{0}\right)$ around $20 \varphi_{0}$ corresponds to a local maximum in $T_{c}$.

The proposal of a defect-unbinding transition, in fact, accounts for the behavior of $R_{0}(T)$ at the local minimum near $20 \varphi_{0}$ where the $T$ dependence is largest. In analogy with zero-field vortex unbinding, we expect the unbound defects to give a contribution $\Delta R \propto \exp \left[-B /\left(T-T_{c}\right)^{1 / 2}\right]$ to $R_{0}$ for $T>T_{c}$. To test this prediction, it is necessary to isolate the effects of the field-induced vortices. We make a crude correction for the nonzero $R_{0}$ at $H=0$ by subtracting this value from the finite$H$ data to yield $\Delta R=R_{0}(H)-R_{0}(0)$. In Fig. 3 we display the $\Delta T=T-T_{c}$ dependence of $\Delta R$ at several $N$ about the minimum at $N=20$. At each $N$, $T_{c}$ was chosen to yield the best straight line in Fig. 3. Below $15 \mathrm{mK}$ the relative subtraction er-

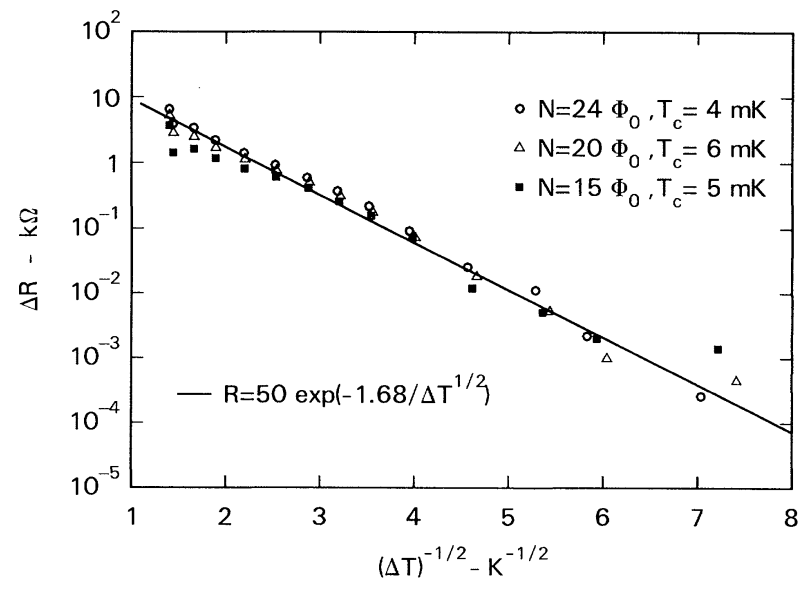

FIG. 3. $\Delta R$ vs $1 / \Delta T^{1 / 2}$ at selected $N$. The straight line is a fit to expected form for a Kosterlitz-Thouless transition. 
rors become too large to yield reliable plots.

Remarkably, all of our data about $N=20$ show the same universal dependence of $\Delta R$ on $\Delta T$. Moreover, a rough fit to the expected form for $\Delta R$ (the solid line in Fig. 3) gives a value for the constant $B \simeq 1.68 \mathrm{~K}^{1 / 2}$ that is consistent with both our measured value for vortex-unbinding at $\boldsymbol{H}=0$ $\left(B \simeq 2.8 \mathrm{~K}^{1 / 2}\right)$ and the theoretical estimate $(B \simeq 3$ $\mathrm{K}^{1 / 2}$ ) for our array. As shown in Fig. 3, the different $R_{0}\left(N \varphi_{0}\right)$ correspond to a field-dependent $T_{c}$ with a maximum $T_{c} \simeq 6 \mathrm{mK}$ at $N=20$. Away from the local minimum $R_{0}(|H|<4 \mathrm{G}$ or $|H|>7.5 \mathrm{G}$ in Fig. 2), the best-fit value for $T_{c} \rightarrow 0$ (even for $N$ $=1$ ). A detailed calculation of $T_{c}$ is, however, beyond the scope of this paper.

In summary, our low- $T$ measurements of the flux mobility in a weakly coupled array demonstrate the great utility of such artificial structures in understanding and probing $2 \mathrm{D}$ physics. Our $R_{0}(H)$ measures the transport properties of interacting particles on a $2 \mathrm{D}$ periodic substrate where the particle density is a continuous function of $H$ and we show the existence of the commensurate $N+\frac{1}{2}$ superlattice. Furthermore, our measurements suggest that defect motion dominates transport at integer numbers of quanta per cell and we introduce the concept of a defectunbinding transition to understand the temperature dependence of $R_{0}\left(N \varphi_{0}\right)$. The surprising $N$ dependence remains, however, unexplained.

We gratefully acknowledge many helpful discussions with S. Faris, A. Davidson, and C. Jayaprakash.

${ }^{1}$ J. M. Kosterlitz and D. J. Thouless, J. Phys. C $\underline{6}$,
1181 (1973).

${ }^{2}$ B. I. Halperin and D. R. Nelson, J. Low Temp. Phys. 36, 599 (1979).

${ }^{3}$ S. Doniach and B. A. Huberman, Phys. Rev. Lett. 42, 1169 (1979); B. A. Huberman and S. Doniach, Phys. Rev. Lett. 43,950 (1979).

${ }^{4}$ D. S. Fisher, Phys. Rev. B 22, 1190 (1980).

${ }^{5}$ D. R. Nelson and B. I. Halperin, Phys. Rev. B $\underline{19}$, 2457 (1979).

${ }^{6}$ A. P. Young, Phys。 Rev. B 19, 1855 (1979).

${ }^{7}$ S. Teitel and C. Jayaprakash, Phys. Rev. B 27, 598 (1983).

${ }^{8}$ C. J. Lobb, D. W. Abraham, and M. Tinkham, Phys. Rev. B 27, 150 (1983).

${ }^{9}$ A. F. Hebard and A. T. Fiory, Phys. Rev. Lett. 44, 291 (1980); A. F. Hebard and J. M. Vandenberg, Phys. Rev. Lett. 44, 50 (1980).

${ }^{10}$ P. A. Bancel and K. E. Gray, Phys. Rev. Lett. 46 , 148 (1981).

${ }^{11}$ S. A. Wolf et a l., Phys. Rev. Lett. 47, 1071 (1981).

${ }^{12}$ A. F. Hebard and A. T. Fiory, in Proceedings of the International Conference on Ordering in $2 D$, edited by

S. K. Sinha (North-Holland, Amsterdam, 1981), p. 181.

${ }^{13}$ Richard F. Voss and Richard A. Webb, Phys. Rev. B 25, 3446 (1982).

${ }^{14}$ D. J. Resnick, J. C. Garland, J. T. Boyd, S. Shoemaker, and R. S. Newrock, Phys. Rev. Lett. $\underline{47}, 1542$ (1981).

${ }^{15}$ D. W. Abraham, C. J. Lobb, M. Tinkham, and T. M. Klapwijk, Phys. Rev. B 26, 5268 (1982)。

${ }^{16}$ A. T. Fiory, A. F. Hebard, and S. Somekh, Appl. Phys. Lett. 32, 73 (1978); O. Daldini, P. Martinoli, J. L. Olsen, and G. Berner, Phys. Rev. Lett. $\underline{32}, 218$ (1974).

${ }^{17}$ We estimate that the pair correlation length increases to the array size at $T \simeq 50 \mathrm{mK}$. At lower $T$, $R_{0}(0)$ fits the expected (Ref. 2) finite-size limited form $R \exp [-E / k T]$ with $R \simeq 35 \Omega$ and $E / k \simeq 70 \mathrm{mK}$ for our sample. 